(A1), (A2), and (A4) imply

$$
\begin{aligned}
S^{e}{ }_{f i}=-i \int d x e^{-i \Delta p x} \bar{u}_{2}(-i) & \\
& \times\left(\epsilon \gamma_{\mu}+\frac{1}{2} i \mu \gamma_{\mu \nu} \Delta p_{\nu}\right) u_{1} A_{\mu}(x),
\end{aligned}
$$

which may also be written as,

$$
\begin{aligned}
S_{f i}{ }_{f i}=-i \int d x e^{-i \Delta p x} & \bar{u}_{2}(-i) \\
& \times\left(\epsilon \gamma_{\mu} A_{\mu}+\frac{1}{2} \mu \gamma_{\mu} \gamma_{\nu}\left(\frac{\partial A_{\mu}}{\partial x_{\nu}}-\frac{\partial A_{\nu}}{\partial x_{\mu}}\right)\right) u_{1} .
\end{aligned}
$$

The invariant functions $\epsilon$ and $\mu$ must depend only on the invariants that can be formed from $p_{1}$ and $p_{2}$, which are $\left(m_{N}\right)^{2}$ and $(\Delta p)^{2}$. This, together with the assumption on the analyticity of $j$ in the neighborhood of $\Delta p_{\mu}=0$, implies that we may write

$$
\begin{aligned}
\boldsymbol{\epsilon} & =\epsilon_{0}-\epsilon_{1}(\Delta p)^{2}+\epsilon_{2}(\Delta p)^{4}-\cdots, \\
\mu & =\mu_{0}-\mu_{1}(\Delta p)^{2}+\mu_{2}(\Delta p)^{4}-\cdots .
\end{aligned}
$$

If these expressions for $\epsilon$ and $\mu$ are subsittuted into the last equation, one readily obtains Eq. (10).

\title{
Causality Conditions and Dispersion Relations. I. Boson Fields*
}

\author{
MaRVin L. Goldberger \\ Institute for Nuclear Studies and Department of Physics, University of Chicago, Chicago, Illinois \\ (Received April 7, 1955)
}

\begin{abstract}
The dispersion relations of Kramers and Kronig as generalized for charged and neutral Bose particles with finite rest mass are derived in a new way using the formalism of quantum field theory. The alternative forms of dispersion relations obtained by making various assumptions on the high-frequency limit of total cross sections are used to obtain information about the high-frequency behavior of the total cross section for the scattering of $\gamma$ rays by electrons.
\end{abstract}

\section{INTRODUCTION}

$I^{N}$ $\mathrm{N}$ two recent papers, ${ }^{1}$ a discussion has been given of the derivation of the Kramers-Kronig dispersion relation for photon propagation from the standpoint of quantum field theory. It was shown in the first of these papers (GGT) that the relation followed from the "causality requirement" that the commutator of two vector potential operators taken at space-like points shall vanish. The derivation was based on the use of perturbation theory and a quite restrictive form of interaction between matter and electromagnetic fields. These two limitations were removed in the second paper (G). Another problem treated in GGT was the dispersion relation for Bose particles with mass. Only a very idealized situation was considered and the discussion was very involved and not very satisfactory. In addition, some erroneous and misleading statements were made in connection with the results. One of the purposes of the present work is to give a simplified and more satisfactory derivation of the generalized dispersion relations for particles with mass based on a general formulation analogous to that used in $\mathrm{G}$.

In Sec. 2, we shall discuss the scattering of bosons with finite mass by a matter system, the exact nature of which is unimportant. We shall use no specific theory to specify the interaction in detail. Only the

\footnotetext{
* Supported in part by a grant from the U. S. Atomic Energy Commission.

${ }^{1}$ Gell-Mann, Goldberger, and Thirring, Phys. Rev. 95, 1612 (1954); M. L. Goldberger, Phys. Rev. 97, 508 (1955).
}

usual assumptions of quantum field theory necessary for the definition of an $S$-matrix are used. Section 3 contains a discussion of the results and a comparison with the work of GGT.

We shall confine our attention in this paper to the scattering of bosons. There are a number of novel features involved in the fermion scattering problem and these will be treated in a separate paper which is being prepared in collaboration with Dr. R. Oehme. We remark only that the dispersion relations derived here do not seem to hold in the case of fermion-fermion scattering processes.

\section{DERIVATION OF DISPERSION RELATIONS}

We consider the scattering of particles of mass $\mu$, described by a real boson field $\phi_{\alpha}(x)$, by a matter system, the exact nature of which need not be specified too closely. (We shall assume, however, that it is distinguishable from the projectile field. The difficulties which arise in the case of identical particle scattering will be discussed in Sec. 3.) The index $\alpha$ may designate an internal degree of freedom of the boson field, such as isotopic spin, polarization, etc. The Heisenberg equation of motion for $\phi_{\alpha}$ will be taken to be

$$
\left(\mu^{2}-\square^{2}\right) \phi_{\alpha}(x)=j_{\alpha}(x),
$$

where the "current," $j_{\alpha}(x)$, will be assumed to depend, perhaps, on $\phi_{\alpha}(x)$, but on $\dot{\phi}_{\alpha}(x)$. (To avoid the latter we do not carry out explicit wave function renormaliza- 
tion; if the above assumption is not correct, or if one wants to do the renormalization at this point, it is simple to generalize the derivations. ${ }^{2}$ We assume that $\phi_{\alpha}$ is an Hermitian operator. We shall furthermore demand that

$$
\left[j_{\alpha}(x), j_{\beta}(y)\right]=0, \quad(x-y)^{2}>0,
$$

which follows from Eq. (2.1) and the requirement that the commutator of two $\phi$ 's taken at space-like points shall vanish. This is our causality condition.

A typical physical situation which could be described by our formalism is the scattering of symmetrical pseudoscalar mesons by nucleons in which case the index $\alpha$ refers to the isotopic spin of the meson field. In the conventional theory, $j_{\alpha}(x)$ is given by

$$
j_{\alpha}(x)=-i g \bar{\psi} \gamma_{5} \tau_{\alpha} \psi+\delta \mu^{2} \phi_{\alpha}-\lambda\left(\phi_{\beta} \phi_{\beta}\right) \phi_{\alpha},
$$

where we have explicitly included the mass and mesonmeson interaction renormalizations.

We now calculate the Feynman matrix element for the process in which a particle of four-momentum $q$, index $\beta$, is scattered into a particle of four-momentum $k$, index $\alpha$, while the matter system goes from $p, \lambda$ to $p^{\prime}, \lambda^{\prime}$. The labels $\lambda, \lambda^{\prime}$ mean all quantum numbers beside the four-momenta, $p, p^{\prime}$ needed to characterize the matter system. This matrix element is given, just as in $\mathrm{G}$, by

$$
\begin{aligned}
& \mathfrak{F}_{\alpha \beta}\left(k, q ; p^{\prime} \lambda^{\prime}, p \lambda\right) \equiv i \int d^{4} x \int d^{4} y e^{-i k \cdot x+i q \cdot y} \\
& \quad \times\left(\mu^{2}-\square_{x^{2}}^{2}\right)\left(\mu^{2}-\square_{y}^{2}\right)\left\langle p^{\prime} \lambda^{\prime}\left|\left(\phi_{\alpha}(x) \phi_{\beta}(y)\right)_{+}\right| p \lambda\right\rangle,
\end{aligned}
$$

where $\left(\phi_{\alpha}(x) \phi_{\beta}(y)\right)_{+}$stands for the familiar $P$-bracket of $\phi_{\alpha}(x)$ and $\phi_{\beta}(y)$. For real particles, $k^{2}=q^{2}=-\mu^{2}$. Carrying out the operations indicated in (2.4), we obtain

$$
\begin{aligned}
& \mathfrak{F}_{\alpha \beta}\left(k, q ; p^{\prime} \lambda^{\prime}, p \lambda\right)=i \int d^{4} x \int d^{4} y e^{-i k \cdot x+i q \cdot y} \\
& \times\left\langle p^{\prime} \lambda^{\prime}\right|\left(j_{\alpha}(x), j_{\beta}(y)\right)_{+}-\delta\left(x_{0}-y_{0}\right) \\
& \times\left[j_{\alpha}(x), \dot{\phi}_{\beta}(y)\right]|p \lambda\rangle .
\end{aligned}
$$

It is convenient at this point to assume that

$$
j_{\alpha}(x)=e^{-i P \cdot x} j_{\alpha}(0) e^{i P \cdot x},
$$

where $P$ is the total momentum operator. [It is sufficient to assume that only the time dependence of $j_{\alpha}$ is given by a relation like (2.6) but the algebra is simplified by the assumption of full translational invariance.] Using Eq. (2.6), we may easily separate from Eq. (2.5) a factor of $(2 \pi)^{4} \delta\left(p^{\prime}+k-p-q\right)$ which expresses the over-all energy momentum conservation. Calling the

2 Wave function renormalization must, of course, be carried out in practice eventually. All of the relations to be derived are homogeneous in the scattering amplitude and a multiplicative factor is of no consequence. coefficient of this factor $F_{\alpha \beta}\left(k, q ; p^{\prime} \lambda^{\prime}, p \lambda\right)$, we have

$$
\begin{aligned}
F_{\alpha \beta}\left(k, q ; p^{\prime} \lambda^{\prime}, p \lambda\right)=i \int & d^{4} x e^{-i k \cdot x}\left\langle p^{\prime} \lambda^{\prime}\right|\left(j_{\alpha}(x) j_{\beta}(0)\right)_{+} \\
& -\delta\left(x_{0}\right)\left[j_{\alpha}(x), \dot{\phi}_{\beta}(0)\right]|p \lambda\rangle
\end{aligned}
$$

where we must remember the restriction $p^{\prime}+k=p+q$. We specialize now to the case of forward, but not necessarily coherent scattering, i.e., we put $p^{\prime}=p, q=k$, but allow $\alpha$ and $\beta$ as well as $\lambda^{\prime}$ and $\lambda$ to be distinct. We write then ${ }^{3}$

$$
\begin{array}{r}
F_{\alpha \beta}\left(k ; \lambda^{\prime}, \lambda\right)=i \int d^{4} x e^{-i k \cdot x}\left\langle p \lambda^{\prime}\right|\left(j_{\alpha}(x) j_{\beta}(0)\right)_{+} \\
-\delta\left(x_{0}\right)\left[j_{\alpha}(x), j_{\beta}(0)\right]|p \lambda\rangle .
\end{array}
$$

The state $|p \lambda\rangle$ of the matter system will be assumed to be stable. Under this circumstance we may define a quantity $M_{\alpha \beta}\left(k ; \lambda^{\prime}, \lambda\right)$ which is fundamentally simpler than $F_{\alpha \beta}\left(k ; \lambda^{\prime}, \lambda\right)$ and which contains all the same information:

$$
\begin{array}{r}
M_{\alpha \beta}\left(k ; \lambda^{\prime}, \lambda\right) \equiv i \int d^{4} x e^{-i k \cdot x}\left\langle p \lambda^{\prime}\right| \eta(x)\left[j_{\alpha}(x), j_{\beta}(0)\right] \\
-\delta\left(x_{0}\right)\left[j_{\alpha}(x), \dot{\phi}_{\beta}(0)\right]|p \lambda\rangle
\end{array}
$$

where $\eta(x)$ is the step function, zero for $x_{0}<0$, unity for $x_{0}>0$. The relation between $F_{\alpha \beta}\left(k ; \lambda^{\prime}, \lambda\right)$ and $M_{\alpha \beta}\left(k ; \lambda^{\prime} \lambda\right)$ is as follows: If the two quantities be written as a sum of a dispersive and an absorptive part, where the division is made according to whether real or virtual intermediate states are involved, one finds

$$
\begin{aligned}
F_{\alpha \beta}\left(k ; \lambda^{\prime}, \lambda\right) & =D_{\alpha \beta}\left(k ; \lambda^{\prime}, \lambda\right)+i \epsilon\left(k_{0}\right) A_{\alpha \beta}\left(k ; \lambda^{\prime}, \lambda\right), \\
M_{\alpha \beta}\left(k ; \lambda^{\prime}, \lambda\right) & =D_{\alpha \beta}\left(k ; \lambda^{\prime}, \lambda\right)+i A_{\alpha \beta}\left(k ; \lambda^{\prime}, \lambda\right) .
\end{aligned}
$$

Thus, for positive frequencies, $k_{0}$, the two quantities are identical, but the absorptive parts differ in sign for negative frequencies. [See the corresponding discussion in GGT, Eqs. (3.12), (3.14), (3.15), (3.16). The notation has been changed slightly: $A \rightarrow \epsilon\left(k_{0}\right) A$.] It should be noted that the quantities $D$ and $A$ are not necessarily real. The great advantage of Eq. (2.9) over Eq. (2.8) for our purposes is that the quantity $\eta(x)\left[j_{\alpha}(x), j_{\beta}(0)\right]$ is a truly retarded function which vanishes not only for $x_{0}<0$ but also for $x_{\mu}^{2}>0$, according to our causality requirement, Eq. (2.2). As we shall see shortly, this is the key to the whole derivation.

It is convenient at this point to choose a definite Lorentz frame in which to evaluate the quantities

${ }^{3}$ The dependence on the momentum $p$ will be suppressed.

${ }^{4}$ We have defined the forward scattering amplitude as the limit of the amplitude for nonforward scattering, as is usually done. Our final formula, however, contains terms which in an actual computation should consequently be dropped, namely, essentially vacuum fluctuation terms in which the state of the matter system is unchanged. We must subtract from the matrix element in Eq. (2.8) $\delta_{\lambda^{\prime} \lambda}$ times the vacuum expectation value of the same operator. This causes no modification of any of our subsequent results, and to save space we shall not write it explicitly. The author is indebted to Professor $\mathrm{X}$. Nambu for this observation. 
appearing in Eq. (2.10). Since both $F_{\alpha \beta}\left(k ; \lambda^{\prime}, \lambda\right)$ and $M_{\alpha \beta}\left(k ; \lambda^{\prime}, \lambda\right)$ are effectively invariants, this entails no loss of generality. We shall choose the system in which $\mathbf{p}$ is zero, the laboratory system. The particular advantage of this system for us is that the $k$ dependence of our quantities $F$ and $M$ is explicitly exhibited in the factor $\exp (-i k \cdot x)$. We may also assume then that the states $|p \lambda\rangle$ has a definite parity.

We wish now to break $M_{\alpha \beta}\left(k ; \lambda^{\prime}, \lambda\right)$ into its dispersive and absorptive parts and then eventually each of these into parts which are even and odd under interchange of $\alpha$ and $\beta$. These operations are facilitated by the observations that

$$
\begin{array}{r}
M_{\alpha \beta}\left(\mathbf{k}, \omega ; \lambda^{\prime}, \lambda\right)=M_{\alpha \beta}\left(-\mathbf{k}, \omega ; \lambda^{\prime}, \lambda\right), \\
\left\langle p \lambda^{\prime}\left|\left[j_{\alpha}(-x), j_{\beta}(0)\right]\right| p \lambda\right\rangle \\
=\left\langle p \lambda^{\prime}\left|\left[j_{\alpha}(0), j_{\beta}(x)\right]\right| p \lambda\right\rangle,
\end{array}
$$

where we have written $k_{0}=\omega$, and, of course, we have $\omega^{2}=\mathbf{k}^{2}+\mu^{2}$. The proof of these statements is elementary: Eq. (2.11a) follows from the observation that the behavior of $\phi_{\alpha}$ (and hence of $j_{\alpha}$ ) under reflections is, with $\mathbf{P}$ the parity operator, $\mathbf{P} \phi_{\alpha}\left(\mathbf{x}, x_{0}\right) \mathbf{P}^{-1}= \pm \phi_{\alpha}\left(-\mathbf{x}, x_{0}\right)$, and that $\mathbf{P}|p \lambda\rangle=\exp (i \chi)|p \lambda\rangle, \chi$ real. Equation (2.11b) follows trivially from the assumed translational invariance, Eq. (2.6). We see from Eq. (2.11a) that $M_{\alpha \beta}\left(\mathbf{k}, \omega ; \lambda^{\prime}, \lambda\right)$ must be an even function of $\mathbf{k}$ and, thus, the $\exp (-i \mathbf{k} \cdot \mathbf{x})$ in Eq. (2.9) may be replaced by $\cos (\mathbf{k} \cdot \mathbf{x})$. The same is true for $F_{\alpha \beta}\left(\mathbf{k}, \omega ; \lambda^{\prime}, \lambda\right)$. These quantities then effectively depend on $\mathbf{k}$ through $\mathbf{k}^{2}$ $=\omega^{2}-\mu^{2}$ and we shall henceforth regard them as functions of $\omega$ only. There appear to be no branch points at $\omega= \pm \mu$, although if there were, they would cause no trouble. Finally, we note the very important relation

$$
M_{\alpha \beta}\left(-\omega ; \lambda^{\prime}, \lambda\right)=\left[M_{\alpha \beta}\left(\omega ; \lambda, \lambda^{\prime}\right)\right]^{*} .
$$

If we regard $M_{\alpha \beta}$ as a matrix in the $\lambda^{\prime}, \lambda$ labels, we may say that changing $\omega$ to $-\omega$ causes $M_{\alpha \beta}$ to go into its Hermitian conjugate. It should be noted that the Feynman amplitude $F_{\alpha \beta}\left(\omega ; \lambda^{\prime}, \lambda\right)$ does not satisfy Eq. (2.11c).

The dispersive and absorptive parts of $M_{\alpha \beta}\left(\omega ; \lambda^{\prime}, \lambda\right)$ are given, respectively, by

$$
\begin{aligned}
D_{\alpha \beta}\left(\omega ; \lambda^{\prime}, \lambda\right) & =\frac{i}{2} \int d^{4} x e^{i \omega x_{0}} \\
\times & \cos (\mathbf{k} \cdot \mathbf{x})\left\langle p \lambda^{\prime}\right| \epsilon(x)\left[j_{\alpha}(x), j_{\beta}(0)\right] \\
& \quad-2 \delta\left(x_{0}\right)\left[j_{\alpha}(x), \dot{\phi}_{\beta}(0)\right]|p \lambda\rangle
\end{aligned}
$$

and

$$
\begin{aligned}
A_{\alpha \beta}\left(\omega ; \lambda^{\prime}, \lambda\right) & =\frac{1}{2} \int d^{4} x e^{i \omega x_{0}} \\
& \times \cos (\mathbf{k} \cdot \mathbf{x})\left\langle p \lambda^{\prime}\left|\left[j_{\alpha}(x), j_{\beta}(0)\right]\right| p \lambda\right\rangle,
\end{aligned}
$$

where we have merely written $\eta(x)=[1+\epsilon(x)] / 2$. Now in all conventional theories, the second term in Eq. (2.12) is independent of $\omega$, since the equal time com- mutator always leads to a factor of $\delta(\mathbf{x})$; furthermore, it is inevitably symmetric in $\alpha$ and $\beta$. We shall assume for simplicity that this circumstance always obtains for the remainder of the discussion. Let us separate Eq. (2.12) and Eq. (2.13) into parts which are symmetric and antisymmetric under interchange of $\alpha$ and $\beta$. With the understanding that the matrix element between the states $\left|p \lambda^{\prime}\right\rangle$ and $|p \lambda\rangle$ will be eventually formed we may write, using $\mathrm{Eq}$. (2.11b),

where

$$
\left[j_{\alpha}(x), j_{\beta}(0)\right]=K_{\alpha \beta}^{(-)}(x)+K_{\alpha \beta}^{(+)}(x),
$$

$$
\begin{aligned}
& K_{\alpha \beta}^{(-)}(x)=\frac{1}{2}\left\{\left[j_{\alpha}(x), j_{\beta}(0)\right]-\left[j_{\alpha}(0), j_{\beta}(x)\right]\right\}, \\
& K_{\alpha \beta}{ }^{(+)}(x)=\frac{1}{2}\left\{\left[j_{\alpha}(x), j_{\beta}(0)\right]+\left[j_{\alpha}(0), j_{\beta}(x)\right]\right\} .
\end{aligned}
$$

Note that $K_{\alpha \beta}^{(-)}(x)$ is an odd function of $x$ whereas $K_{\alpha \beta}{ }^{(+)}(x)$ is even. Furthermore,

$$
\begin{aligned}
& K_{\alpha \beta}{ }^{(-)}(x)=K_{\beta \alpha}{ }^{(-)}(x), \\
& K_{\alpha \beta}{ }^{(+)}(x)=-K_{\beta \alpha}{ }^{(+)}(x) .
\end{aligned}
$$

By using Eqs. (2.15) and (2.16), the separation of Eqs. (2.12) and (2.13) into symmetric and antisymmetric parts is easily carried out. We write

$$
\begin{aligned}
& D_{\alpha \beta}\left(\omega ; \lambda^{\prime}, \lambda\right)=D_{\alpha \beta}{ }^{(1)}\left(\omega ; \lambda^{\prime}, \lambda\right)+i D_{\alpha \beta}{ }^{(2)}\left(\omega ; \lambda^{\prime}, \lambda\right), \\
& A_{\alpha \beta}\left(\omega ; \lambda^{\prime}, \lambda\right)=A_{\alpha \beta}{ }^{(1)}\left(\omega ; \lambda^{\prime}, \lambda\right)+i A_{\alpha \beta}{ }^{(2)}\left(\omega ; \lambda^{\prime}, \lambda\right),
\end{aligned}
$$

where

$$
\begin{aligned}
D_{\alpha \beta}{ }^{(1)}\left(\omega ; \lambda^{\prime}, \lambda\right)= & \frac{i}{2} \int d^{4} x \cos \mathbf{k} \cdot \mathbf{x} \\
\times & \cos \omega x_{0}\left\langle p \lambda^{\prime}\right| \epsilon(x) K_{\alpha \beta}{ }^{(-)}(x) \\
& -2 \delta\left(x_{0}\right)\left[j_{\alpha}(x), \dot{\phi}_{\beta}(0)\right]|p \lambda\rangle, \\
D_{\alpha \beta}{ }^{(2)}\left(\omega ; \lambda^{\prime}, \lambda\right)= & \frac{i}{2} \int d^{4} x \cos \mathbf{k} \cdot \mathbf{x} \\
& \times \sin \omega x_{0}\left\langle p \lambda^{\prime}\left|\epsilon(x) K_{\alpha \beta}{ }^{(+)}(x)\right| p \lambda\right\rangle, \\
A_{\alpha \beta}{ }^{(1)}\left(\omega ; \lambda^{\prime}, \lambda\right)= & \frac{i}{2} \int d^{4} x \cos \mathbf{k} \cdot \mathbf{x} \\
& \times \sin \omega x_{0}\left\langle p \lambda^{\prime}\left|K_{\alpha \beta}^{(-)}(x)\right| p \lambda\right\rangle, \\
A_{\alpha \beta}{ }^{(2)}\left(\omega ; \lambda^{\prime}, \lambda\right)= & -\frac{i}{2} \int d^{4} x \cos \mathbf{k} \cdot \mathbf{x} \\
& \times \cos \omega x_{0}\left\langle p \lambda^{\prime}\left|K_{\alpha \beta}{ }^{(+)}(x)\right| p \lambda\right\rangle .
\end{aligned}
$$

By construction, the quantities labeled with superscript 1 are symmetric in $\alpha$ and $\beta$, while those labeled 2 are antisymmetric. All four quantities are hermitian matrices in the $\lambda^{\prime}, \lambda$ space. Note that regarded as functions of the frequency, $\omega, D^{(1)}$ and $A^{(2)}$ are even functions whereas $D^{(2)}$ and $A^{(1)}$ are odd. If $\lambda^{\prime}=\lambda$, all four quantities are real. Finally, we see that the integrations over $x_{0}$ may be restricted to positive values only.

There is one further step which must be taken before we proceed to the derivation of the dispersion relations. 
We must investigate the nature of whatever singularities might be expected for real values of $\omega$ for the function $A_{\alpha \beta}\left(\omega ; \lambda^{\prime}, \lambda\right)$. To simplify the writing, we will not at this point separate $A$ into its two parts as we did previously. We simply expand the matrix element in Eq. (2.13) into a complete set of states for the interacting systems and obtain, after carrying out the $x_{0}$ integration,

$$
\begin{aligned}
A_{\alpha \beta}\left(\omega ; \lambda^{\prime}, \lambda\right)= & \pi \sum_{n}\left[f_{\alpha \beta}\left(\omega, n ; \lambda^{\prime}, \lambda\right) \delta\left(E_{p}-E_{n}+\omega\right)\right. \\
& \left.-f_{\alpha \beta} *\left(\omega, n ; \lambda, \lambda^{\prime}\right) \delta\left(E_{p}-E_{n}-\omega\right)\right],
\end{aligned}
$$

where

$$
\begin{aligned}
f_{\alpha \beta}\left(\omega, n ; \lambda^{\prime}, \lambda\right)=\int & d^{3} x e^{-i \mathbf{k} \cdot \mathbf{x}} \\
& \times\left\langle p \lambda^{\prime}\left|j_{\alpha}(\mathbf{x})\right| n\right\rangle\left\langle n\left|j_{\beta}(0)\right| p \lambda\right\rangle .
\end{aligned}
$$

We note that unless there are discrete bound states of energy $E_{n}=E_{p}+\mu-B_{n}, B_{n}>0, A_{\alpha \beta}\left(\omega ; \lambda^{\prime}, \lambda\right)$ is zero for $|\omega|<\mu$. This point was overlooked in GGT and was pointed out to the author by F. Low and Y. Nambu, independently. It has been further discussed by Karplus and Ruderman in a recent paper. ${ }^{5}$ It is clear from Eq. (2.23) that there will be branch points for values of $|\omega|>\mu$ corresponding to the thresholds for real reactions.

Consider now the quantity $I_{\alpha \beta}\left(\omega, \omega_{0} ; \lambda^{\prime}, \lambda\right)$ defined by

$$
\begin{aligned}
& I_{\alpha \beta}\left(\omega, \omega_{0} ; \lambda^{\prime}, \lambda\right) \equiv \frac{\omega^{2}-\omega_{0}^{2}}{\pi} \int_{-\infty}^{\infty} d \omega^{\prime} \\
& \times \frac{A_{\alpha \beta}{ }^{(1)}\left(\omega^{\prime} ; \lambda^{\prime}, \lambda\right)+i A_{\alpha \beta}{ }^{(2)}\left(\omega^{\prime} ; \lambda^{\prime}, \lambda\right)}{\left(\omega^{\prime}-\omega_{0}\right)\left(\omega^{2}-\omega^{2}\right)} .
\end{aligned}
$$

The integrations over the singularities are to be taken in sense of Cauchy principal values and the branch points mentioned above are to be detoured by small semicircles into the upper half plane. From the nature of the $\omega$ dependence in the neighborhood of any such threshold point, it is clear that as the semicircles are shrunk to zero, they make no contribution to the integral. The only role played by the branch points is to determine the phase of $A_{\alpha \beta}\left(\omega^{\prime} ; \lambda^{\prime}, \lambda\right)$ on either side of them. The frequencies $\omega, \omega_{0}$ are arbitrary, but we shall take them to be greater than or equal to $\mu$. We shall now substitute for $A_{\alpha \beta}\left(\omega^{\prime} ; \lambda^{\prime}, \lambda\right)$ its value as given by Eqs. (2.18), (2.21), and (2.22), and interchange the order of integration. Recall now that the space-time integrations in these expressions for the absorptive parts extend over the interior and surface of the future light cone. As long as $x_{0}>|\mathbf{x}|$, the proposed interchange may be probably justified without too great difficulty, since the contributions to $A_{\alpha \beta}\left(\omega^{\prime} ; \lambda^{\prime}, \lambda\right)$ from these regions approach zero for large $\omega$. (This assumes that the quantities $\left\langle p \lambda^{\prime}\left|K_{\alpha \beta}{ }^{\left({ }^{ \pm}\right)}(x)\right| p \lambda\right\rangle$ are reasonably well behaved, which is an assumption which underlies all field theoretic calculations. If this is so, then since $x_{0}>|\mathbf{x}|$, we always have an oscillatory integrand which will make the integral go to zero as $|\omega| \rightarrow \infty$.) The con-

\footnotetext{
${ }^{5}$ R. Karplus and M. Ruderman, Phys. Rev. 98, 771 (1955).
}

tributions from the light cone itself cannot be neglected since we must be prepared for very singular behavior of our matrix element, such as being proportional to $\delta\left(x^{2}\right)$ or even derivatives thereof. It is, of course, the high-frequency behavior which will be determined from the matrix elements on the light cone, and we must assume that we have supplied a sufficient number of inverse powers of $\omega$ to make our procedure legitimate. We shall return to this point later in the discussion.

If the above outlined steps are carried out, we obtain,

$$
\begin{aligned}
& I_{\alpha \beta}\left(\omega, \omega_{0} ; \lambda^{\prime}, \lambda\right)=i \int_{0}^{\infty} d x_{0} \int d^{3} x \\
& \times\left[\left\langle p \lambda^{\prime}\left|K_{\alpha \beta}^{(-)}(x)\right| p \lambda\right\rangle J^{(1)}\left(x ; \omega, \omega_{0}\right)\right. \\
& \left.+\left\langle p \lambda^{\prime}\left|K_{\alpha \beta}{ }^{(+)}(x)\right| p \lambda\right\rangle i J^{(2)}\left(x ; \omega, \omega_{0}\right)\right] \text {, } \\
& \text { where } \\
& J^{(1)}\left(x ; \omega, \omega_{0}\right)=\frac{\omega^{2}-\omega_{0}^{2}}{\pi} \int_{-\infty}^{\infty} d \omega^{\prime} \frac{\omega^{\prime} \sin \omega^{\prime} x_{0} \cos \mathbf{k}^{\prime} \cdot \mathbf{x}}{\left(\omega^{\prime 2}-\omega_{0}^{2}\right)\left(\omega^{\prime 2}-\omega^{2}\right)}, \\
& J^{(2)}\left(x ; \omega, \omega_{0}\right)=-\frac{\omega^{2}-\omega_{0}^{2}}{\pi} \int_{-\infty}^{\infty} d \omega^{\prime} \\
& \times \frac{\cos \omega^{\prime} x_{0} \cos \mathbf{k}^{\prime} \cdot \mathbf{x}}{\left(\omega^{\prime 2}-\omega_{0}^{2}\right)\left(\omega^{\prime 2}-\omega^{2}\right)} .
\end{aligned}
$$

These integrals may be evaluated by elementary contour integration following the replacement of $\sin \omega x_{0}$ by $-i \exp \left(i \omega x_{0}\right)$ and $\cos \omega x_{0}$ by $\exp \left(i \omega x_{0}\right)$, since for $x_{0}>|\mathbf{x}|$, these factors dominate the integrands, and even for $x_{0}=|\mathbf{x}|, \exp \left(i \omega x_{0}\right) \cos k^{\prime} \cdot x$ is bounded, and we have supplied enough inverse powers of $\omega$ that the usual infinite semicircle in the upper half $\omega$ plane makes no contribution. We obtain then

$$
\begin{aligned}
J^{(1)}\left(x ; \omega, \omega_{0}\right) & =\cos \omega x_{0} \cos \mathbf{k} \cdot \mathbf{x}-\cos \omega_{0} x_{0} \cos \mathbf{k}_{0} \cdot \mathbf{x} \\
J^{(2)}\left(x ; \omega, \omega_{0}\right) & =\left(\omega_{0} / \omega\right) \sin \omega x_{0} \cos \mathbf{k} \cdot \mathbf{x} \\
& -\sin \omega_{0} x_{0} \cos \mathbf{k}_{0} \cdot \mathbf{x} .
\end{aligned}
$$

Substituting these equations into Eq. (2.26) and comparing with Eqs. (2.19) and (2.20), we find

$$
\begin{gathered}
I_{\alpha \beta}\left(\omega, \omega_{0} ; \lambda^{\prime}, \lambda\right)=D_{\alpha \beta}{ }^{(1)}\left(\omega ; \lambda^{\prime}, \lambda\right)-D_{\alpha \beta}{ }^{(1)}\left(\omega_{0} ; \lambda^{\prime}, \lambda\right) \\
\quad+i\left[\left(\omega_{0} / \omega\right) D_{\alpha \beta}{ }^{(2)}\left(\omega ; \lambda^{\prime}, \lambda\right)-D_{\alpha \beta}{ }^{(2)}\left(\omega_{0} ; \lambda^{\prime}, \lambda\right)\right],
\end{gathered}
$$

where we have assumed that the second term in Eq. (2.19) is a constant, independent of frequency. Since the quantities with superscript 1 (2) are symmetric (antisymmetric) under interchange of $\alpha$ and $\beta$, we may separate Eq. (2.31) into two distinct parts:

$$
\begin{aligned}
& D_{\alpha \beta^{(1)}}\left(\omega ; \lambda^{\prime}, \lambda\right)-D_{\alpha \beta}{ }^{(1)}\left(\omega_{0} ; \lambda^{\prime}, \lambda\right) \\
& =\frac{2\left(\omega^{2}-\omega_{0}^{2}\right)}{\pi} \int_{0}^{\infty} d \omega^{\prime} \omega^{\prime} \frac{A_{\alpha \beta^{(1)}}\left(\omega^{\prime} ; \lambda^{\prime}, \lambda\right)}{\left(\omega^{\prime 2}-\omega_{0}^{2}\right)\left(\omega^{\prime 2}-\omega^{2}\right)} \\
& D_{\alpha \beta}{ }^{(2)}\left(\omega ; \lambda^{\prime}, \lambda\right)-\frac{\omega}{\omega_{0}} D_{\alpha \beta^{(2)}}\left(\omega_{0} ; \lambda^{\prime}, \lambda\right) \\
& =\frac{2 \omega\left(\omega^{2}-\omega_{0}^{2}\right)}{\pi} \int_{0}^{\infty} d \omega^{\prime} \frac{A_{\alpha \beta}{ }^{(2)}\left(\omega^{\prime} ; \lambda^{\prime}, \lambda\right)}{\left(\omega^{\prime 2}-\omega_{0}{ }^{2}\right)\left(\omega^{\prime 2}-\omega^{2}\right)}
\end{aligned}
$$


We have used the fact that $A_{\alpha \beta}{ }^{(1)}$ is an even function of $\omega$ while $A_{\alpha \beta}{ }^{(2)}$ is odd in rewriting the definition of $I_{\alpha \beta}$, Eq. (2.25). These are the generalized dispersion relations appropriate for particles with mass. They correspond to Eqs. (5.13) of GGT, and in fact coincide with them in the limit $\mu \rightarrow 0$.

Our final task is to express the integrals appearing in Eqs. (2.32) and (2.33) in terms of physical quantities in so far as it is possible. We recall that our fundamental quantity, $M_{\alpha \beta}\left(\omega ; \lambda^{\prime}, \lambda\right)$, becomes identical with the Feynman matrix element, $F_{\alpha \beta}\left(\omega ; \lambda^{\prime}, \lambda\right)$, for $\omega>0$. The relation between this quantity and the true forward scattering amplitude for elastic scattering, $T_{\alpha \beta}\left(\omega ; \lambda^{\prime}, \lambda\right)$, in general depends the choice of a Lorentz frame. For any two particle scattering process, $T_{\alpha \beta}\left(\omega ; \lambda^{\prime}, \lambda\right)=F_{\alpha \beta}\left(\omega^{\prime} ; \lambda^{\prime}, \lambda\right)$ for the laboratory system in which the target is initially at rest, to within a constant factor. ${ }^{6}$ In virtue of the fact that $T_{\alpha \beta}\left(\omega ; \lambda^{\prime}, \lambda\right) /|\mathbf{k}|$ is an invariant we may easily convert our forward scattering amplitudes to the center-of-mass system: $\left[T_{\alpha \beta}\right]_{\text {lab }}$ $=(W / M)\left[T_{\alpha \beta}\right]_{\text {c.m. }}$., where $W$ is the total energy in the center-of-mass frame and $M$ is the mass of the target. We know also that for $\omega>\mu$, the absorptive parts of $T_{\alpha \beta}$ are related to what we may loosely refer to as total cross sections by the so-called optical theorem. In general only the portion $A_{\alpha \beta}{ }^{(1)}(\omega ; \lambda, \lambda)$ which is symmetric in $\alpha$ and $\beta$ is related to a true physical cross section but under some circumstances $A_{\alpha \beta}{ }^{(2)}(\omega ; \lambda, \lambda)$ may also be so identified. For the energy interval $0 \leq \omega<\mu$, we shall use the expression Eq. (2.23) for $A_{\alpha \beta}\left(\omega ; \lambda^{\prime}, \lambda\right)$, appropriately broken into its symmetric and antisymmetric parts:

$$
\begin{aligned}
A_{\alpha \beta}{ }^{(1)}\left(\omega ; \lambda^{\prime}, \lambda\right) & =\pi \sum_{n}\left\{f_{\alpha \beta}{ }^{(+)}\left(\omega, n ; \lambda^{\prime}, \lambda\right) \delta\left(E_{p}-E_{n}+\omega\right)\right. \\
& \left.-f_{\alpha \beta}(+) *\left(\omega, n ; \lambda^{\prime}, \lambda\right) \delta\left(E_{p}-E_{n}-\omega\right)\right\}, \\
A_{\alpha \beta}{ }^{(2)}\left(\omega ; \lambda^{\prime}, \lambda\right) & =\pi \sum_{n}\left\{f_{\alpha \beta}^{(-)}\left(\omega, n ; \lambda^{\prime}, \lambda\right) \delta\left(E_{p}-E_{n}+\omega\right)\right. \\
& \left.+f_{\alpha \beta}(-) *\left(\omega, n ; \lambda^{\prime}, \lambda\right) \delta\left(E_{p}-E_{n}-\omega\right)\right\},
\end{aligned}
$$

where

$$
\begin{aligned}
f_{\alpha \beta}{ }^{(+)}\left(\omega, n ; \lambda^{\prime}, \lambda\right) & \\
= & {\left[f_{\alpha \beta}\left(\omega, n ; \lambda^{\prime} \lambda\right)+f_{\beta \alpha}\left(\omega, n ; \lambda^{\prime}, \lambda\right)\right] / 2, } \\
f_{\alpha \beta}{ }^{(-)}\left(\omega, n ; \lambda^{\prime}, \lambda\right) & \\
= & {\left[f_{\alpha \beta}\left(\omega, n ; \lambda^{\prime}, \lambda\right)-f_{\beta \alpha}\left(\omega, n ; \lambda^{\prime}, \lambda\right)\right] / 2 i . }
\end{aligned}
$$

We now write Eqs. (2.32) and (2.33) in terms of total cross sections and bound state contributions by using for $A_{\alpha \beta}{ }^{(1)}$ and $A_{\alpha \beta}{ }^{(2)}$ the above expressions for $0<\omega<\mu$ and for $\omega>\mu$, we define two "cross sections," $\sigma_{\alpha \beta}{ }^{(1)}(\omega, \lambda)$ and $\sigma_{\alpha \beta}{ }^{(2)}(\omega, \lambda)$, by

$$
\begin{aligned}
& \sigma_{\alpha \beta}{ }^{(1)}(\omega ; \lambda)=(4 \pi / k) A_{\alpha \beta}{ }^{(1)}(\omega ; \lambda, \lambda), \\
& \sigma_{\alpha \beta}{ }^{(2)}(\omega ; \lambda)=(4 \pi / k) A_{\alpha \beta}{ }^{(2)}(\omega ; \lambda, \lambda) .
\end{aligned}
$$

We have set $\lambda^{\prime}=\lambda$, for it is only when one has not changed the state of the scatterer that one has the co-

\footnotetext{
${ }^{6}$ In meson-nucleon scattering, for example, $F_{\alpha \beta}=4 \pi T_{\alpha \beta}$ if we use Heaviside units for the meson field; with Gaussian units, $F_{\alpha \beta}=T_{\alpha \beta}$.
}

herence necessary for the optical theorem to hold. We have also written $|\mathbf{k}|=k, k^{2}=\omega^{2}-\mu^{2}$. It is convenient to introduce the wave number as the variable of integration and we find, using the same letter to designate now fundtions of $k$ as we had previously used for functions of $\omega$, the results

$$
\begin{aligned}
& D_{\alpha \beta}{ }^{(1)}(k ; \lambda)-D_{\alpha \beta}{ }^{(1)}\left(k_{0} ; \lambda\right)=\frac{k^{2}}{2 \pi^{2}} \int_{0}^{\infty} d k^{\prime} \frac{\sigma_{\alpha \beta}{ }^{(1)}\left(k^{\prime} ; \lambda\right)}{k^{\prime 2}-k^{2}} \\
& -\frac{k_{0}^{2}}{2 \pi^{2}} \int_{0}^{\infty} d k^{\prime} \frac{\sigma_{\alpha \beta}^{(1)}\left(k^{\prime} ; \lambda\right)}{k^{\prime 2}-k_{0}{ }^{2}}+2 \sum_{n}\left(E_{n}-E_{p}\right) \\
& \times f_{\alpha \beta}{ }^{(+)}\left(E_{n}-E_{p}, n ; \lambda\right)\left[\frac{1}{\left(E_{n}-E_{p}\right)^{2}-k^{2}-\mu^{2}}\right. \\
& \left.-\frac{1}{\left(E_{n}-E_{p}\right)^{2}-k_{0}^{2}-\mu^{2}}\right] \text {, } \\
& D_{\alpha \beta}{ }^{(2)}(k ; \lambda)-\frac{\omega}{\omega_{0}} D_{\alpha \beta}{ }^{(2)}\left(k_{0} ; \lambda\right)=\frac{\omega k^{2}}{2 \pi^{2}} \int_{0}^{\infty} \frac{d k^{\prime}}{\omega^{\prime}} \frac{\sigma_{\alpha \beta}{ }^{(2)}\left(k^{\prime} ; \lambda\right)}{k^{\prime 2}-k^{2}} \\
& -\frac{\omega k_{0}^{2}}{2 \pi^{2}} \int_{0}^{\infty} \frac{d k^{\prime}}{\omega^{\prime}} \frac{\sigma_{\alpha \beta}{ }^{(2)}\left(k^{\prime} ; \lambda\right)}{k^{\prime 2}-k_{0}{ }^{2}}+2 \omega \sum_{n} f_{\alpha \beta}^{(-)}\left(E_{n}-E_{p}, n ; \lambda\right) \\
& \times\left[\frac{1}{\left(E_{n}-E_{p}\right)^{2}-k^{2}-\mu^{2}}-\frac{1}{\left(E_{n}-E_{p}\right)^{2}-k_{0}{ }^{2}-\mu^{2}}\right] .
\end{aligned}
$$

The sums extend over those states $|n\rangle$ for which $E_{n}-E_{p} \leq \mu{ }^{7}$ The most natural reference energy is $k_{0}=0$, in which case our formulas take their final form:

$$
\begin{aligned}
& D_{\alpha \beta}{ }^{(1)}(k ; \lambda)-D_{\alpha \beta^{(1)}}(0 ; \lambda)=\frac{k^{2}}{2 \pi^{2}} \int_{0}^{\infty} d k^{\prime} \frac{\sigma_{\alpha \beta^{(1)}}\left(k^{\prime} ; \lambda\right)}{k^{\prime 2}-k^{2}} \\
& +2 \sum_{n}\left(E_{n}-E_{p}\right) f_{\alpha \beta}^{(+)}\left(E_{n}-E_{p}, n ; \lambda\right) \\
& \times\left[\frac{1}{\left(E_{n}-E_{p}\right)^{2}-k^{2}-\mu^{2}}-\frac{1}{\left(E_{n}-E_{p}\right)^{2}-\mu^{2}}\right], \\
& D_{\alpha \beta}{ }^{(2)}(k ; \lambda)-{ }_{\mu}^{\omega} D_{\alpha \beta}{ }^{(2)}(0 ; \lambda)=\frac{k^{2} \omega}{2 \pi^{2}} \int_{0}^{\infty} \frac{d k^{\prime}}{\omega^{\prime}} \frac{\sigma_{\alpha \beta}{ }^{(2)}\left(k^{\prime}\right)}{k^{\prime 2}-k^{2}} \\
& +2 \omega \sum_{n} f_{\alpha \beta}{ }^{(-)}\left(E_{n}-E_{p}, n ; \lambda\right) \\
& \times\left[\frac{1}{\left(E_{n}-E_{p}\right)^{2}-k^{2}-\mu^{2}}-\frac{1}{\left(E_{n}-E_{p}\right)^{2}-\mu^{2}}\right] .
\end{aligned}
$$

${ }^{7}$ We have included in the bound state contribution only terms arising from the singularities associated with the first $\delta$ function Eq. (2.34). These correspond to true discrete bound states of energy $M<E_{n}<M+\mu$. It is also possible for the argument of the second $\delta$ function to vanish and in fact in the case of mesonnucleon scattering it is only this second $\delta$ function which makes a "bound state" contribution. It is not a true bound state, of course; however when the energy of the meson is continued below the rest energy, and the state $|n\rangle$ corresponds to a one-nucleon state, we indeed find a singularity in the scattering amplitude. This point is discussed in the paper on meson-nucleon scattering which immediately follows this one. 
A typical application of these generalized KramersKronig relations is to the problem of meson nucleon scattering. We shall treat this case in detail in a separate paper, so at this time we merely mention the physical significance of $D^{(1)}, D^{(2)}, \sigma^{(1)}$ and $\sigma^{(2)}: D^{(1)}\left(D^{(2)}\right)$ is one half the sum (difference) of the dispersive forward amplitudes for $\pi^{-}+p$ and $\pi^{+}+p$ (ordinary) scattering and consequently $\sigma^{(1)}\left(\sigma^{(2)}\right)$ is one half the sum (difference) of the total $\pi^{(-)}$and $\pi^{(+)}$cross sections for all processes. These results follow from the fact that the total forward scattering amplitude, regarded as a matrix in nucleon charge space, may be written as

$$
T_{\alpha \beta}(\omega)=\delta_{\alpha \beta} T^{(1)}(\omega)+i \epsilon_{\alpha \beta \gamma} \tau_{\gamma} T^{(2)}(\omega),
$$

assuming, of course, symmetrical theory, and

$$
T^{(j)}(\omega)=D^{(j)}(\omega)+i \epsilon(\omega) A^{i}(\omega), \quad j=1,2 .
$$

There is a bound state corresponding to the neutron whose contribution may be calculated from meson theory almost exactly (to within terms of order $\left.(\mu / M)^{2}\right)$; numerically, it is a very important effect.

\section{DISCUSSION AND COMPARISON WITH GGT}

The actual simplicity of the derivation of the generalized dispersion relations, Eqs. (2.40) and (2.41) has probably been obscured by the algebraic details. For this reason it is perhaps worthwhile to summarize the procedure. We first observed that the Feynman matrix element describing the scattering event could be replaced by a quantity which was the Fourier transform of a function which vanished everywhere except inside the future light cone. This new quantity was divided into two parts one of which satisfied the relation $M^{(1)}(-\omega ; \lambda)=\left[M^{(1)}(\omega ; \lambda)\right]^{*}$ and the other satisfied $M^{(2)}(-\omega ; \lambda)=-\left[M^{(2)}(\omega ; \lambda)\right]^{*} .{ }^{8}$ An integral operation, Eq. (2.25), was applied to the absorptive part of $M^{(1)}$ $+i M^{(2)}$ and the two dispersion relations (2.32) and (2.33) emerged. The remainder of the task was to express these results in terms of physical quantities. There are, however, a number of points concerning the final relations which should be discussed further.

The reason for the special role played by the forward scattering amplitude would appear to be the fact that the entire $k$-dependence, as given, say, by Eq. (2.9) occurs in the explicit $\exp (-i k \cdot x)$, whereas if $p^{\prime} \neq p$, but instead $p^{\prime}=p+q-k$, the $k$ dependence becomes much more complicated. In this connection, it should be noted that the dispersion relations which we have derived cannot necessarily be expected to hold without modification for identical particle scattering processes, since the forward amplitude for such events is a linear combination of forward and backward scatterings for nonidentical particles and the backward amplitude does

\footnotetext{
${ }^{8}$ Note that it is $M$, rather than the Feynman amplitude (which
is essentially the same as the scattering amplitude) which has these simple properties and which is the fundamental quantity for our purposes.
}

not satisfy the normal relations. This point will be taken up in more detail in connection with fermionfermion scattering in another paper. The problem of finding dispersion relations for other than forward scattering is being studied further.

In this paper we avoided explicit mention of the behavior of the forward scattering amplitude or rather our quantity $M_{\alpha \beta}\left(\omega ; \lambda^{\prime}, \lambda\right)$, in the complex $\omega$ plane. The use of complex variable theory does not appear to add anything to our understanding, although it is certainly true that a sufficient condition for the existence of our general dispersion relations is that $M_{\alpha \beta}\left(\omega ; \lambda^{\prime}, \lambda\right)$ be the boundary value of a function which is analytic in the upper half plane, together with certain boundedness requirements. Our "boundedness requirement" had to do with the legitimacy of the interchanging of certain orders of integration. Because of the fact that principal value integrals are rather singular, the conventional theorems on such matters are not immediately applicable. However reference to our integral operation, Eq. (2.25), shows that at the point where the operation is really singular, namely if $\omega=\omega_{0}$, we have the factor $\left(\omega^{2}-\omega_{0}^{2}\right)$ in front which is then zero, and thus the singularity is removed. The remaining troublesome point that is involved is the behavior of the scattering amplitude for large frequencies. That this is indeed the case was borne out by examples in which the results of the two orders of integration were compared. Our particular form of the dispersion relations, Eqs. (2.40) and (2.41) would appear to be valid provided $\sigma_{\alpha \beta^{(1)}}(\omega ; \lambda) / \omega$ and $\sigma_{\alpha \beta^{(2)}}(\omega ; \lambda) / \omega^{2}$ tend to zero for $\omega \rightarrow \infty$. The behavior of scattering amplitudes at high energies is of central importance in field theory and it should be clearly recognized that the precise form of any dispersion relation depends on this (unknown) high-energy behavior. Violations of the dispersion relations as given might perhaps be interpreted as an error in the assumed behavior, although it is certainly possible that it means that our original causality requirement, Eq. (2.2), has perhaps broken down at small distances.

It is interesting to see what happens if we make our high-frequency assumption more stringent. For simplicity we confine our remarks to the completely coherent amplitude, i.e., $\alpha=\beta, \lambda^{\prime}, \lambda$, and assume that there are no bound states. Further, assume that the second (and usually constant) term in Eq. (2.9) is absent. A physical model which satisfies these conditions is provided by ordinary quantum electrodynamics. We shall assume that $\boldsymbol{S}^{\infty} d \omega \sigma_{\alpha \alpha}{ }^{(1)}(\omega ; \lambda)$ is bounded, i.e., $\omega \sigma_{\alpha \alpha}{ }^{(1)}(\omega ; \lambda) \rightarrow 0$ as $\omega \rightarrow \infty$. Under these circumstances we may consider, instead of Eq. (2.25), a quantity $I_{\alpha \alpha}{ }^{\prime}$, defined by

$$
I_{\alpha \alpha^{\prime}}\left(\omega, \omega_{0} ; \lambda\right) \equiv \frac{\omega^{2}-\omega_{0}^{2}}{\pi} \int_{-\infty}^{\infty} d \omega^{\prime} \frac{\omega^{\prime 3} A_{\alpha \alpha^{(1)}}\left(\omega^{\prime} ; \lambda\right)}{\left(\omega^{\prime 2}-\omega_{0}^{2}\right)\left(\omega^{\prime 2}-\omega^{2}\right)} .
$$


This quantity is evaluated, as before, with the result

$$
I_{\alpha \alpha}{ }^{\prime}\left(\omega, \omega_{0} ; \lambda\right)=\omega^{2} D_{\alpha \alpha}{ }^{(1)}(\omega ; \lambda)-\omega_{0}^{2} D_{\alpha \alpha}^{(1)}\left(\omega_{0} ; \lambda_{0}\right),
$$

and following the same steps which led to Eqs. (2.40) and (2.41), we find

$$
\begin{array}{r}
D_{\alpha \alpha}^{(1)}(k ; \lambda)-\frac{\mu^{2}}{\omega^{2}} D_{\alpha \alpha}(1)(0 ; \lambda)-\frac{1}{2 \pi^{2}} \frac{k^{2}}{\omega^{2}} \int_{0}^{\infty} d k \sigma_{\alpha \alpha}(1)(k ; \lambda) \\
=\frac{k^{2}}{2 \pi^{2}} \int_{0}^{\infty} d k^{\prime} \frac{\sigma_{\alpha \alpha^{(1)}}(k ; \lambda)}{k^{\prime 2}-k^{2}} .
\end{array}
$$

Evidently, if (3.3) is valid, so is (2.40) and comparison of the two shows that

$$
D_{\alpha \alpha}^{(1)}(0 ; \lambda)=\frac{1}{2 \pi^{2}} \int_{0}^{\infty} d k \sigma_{\alpha \alpha^{(1)}}(k ; \lambda)
$$

Consider now the scattering of photons by free electrons. We know that $D_{\alpha \alpha}{ }^{(1)}(0 ; \lambda)=-e^{2} / m$, where $e$ and $m$ are the physical charge and mass of the electron. Thus, Eq. (3.4) cannot be satisfied and we must conclude that the assumed existence of $\int^{\infty} d k \sigma_{\alpha \alpha}{ }^{(1)}(k ; \lambda)$ is incorrect. All indications from perturbation theory lead one to believe that the integral diverges, nevertheless it is interesting that a rigorous demonstration of the fact may be given. It should be noted that although we have proved that Eq. (3.3) is not applicable in quantum electrodynamics, we have not established the correctness of Eq. (2.40).

Before concluding, we must make a few remarks on the relation between the results of the present paper and those of GGT. For the case of photon scattering, they are identical. However, for the case of particles with mass, aside from the bound states which were assumed absent by them, their formula, namely,

$$
\begin{aligned}
D_{\alpha \alpha^{(1)}}(\omega ; \lambda)-D_{\alpha \alpha}{ }^{(1)}(\omega & =0 ; \lambda) \\
& =\frac{2 \omega^{2}}{\pi} \int_{0}^{\infty} d \omega^{\prime} \frac{A_{\alpha \alpha^{(1)}}\left(\omega^{\prime} ; \lambda\right)}{\omega^{\prime}\left(\omega^{\prime 2}-\omega^{2}\right)},
\end{aligned}
$$

corresponds to choosing the $\omega_{0}$ of the previous section equal to zero, instead of $\mu$, as we have done. As has been remarked earlier, if there are no bound states, the lower limit of the integral should be set equal to $\mu$. Then, changing to the wave number as variable, we may rewrite Eq. (3.5) as

$$
\begin{aligned}
D_{\alpha \alpha^{(1)}}(k ; \lambda)-D_{\alpha \alpha^{(1)}}\left(k^{2}=-\mu^{2} ; \lambda\right) \\
\quad=\frac{k^{2}}{2 \pi^{2}} \int_{0}^{\infty} d k^{\prime} \frac{\sigma_{\alpha \alpha^{(1)}}\left(k^{\prime}\right)}{k^{\prime 2}-k^{2}}+\frac{\mu^{2}}{2 \pi^{2}} \int_{0}^{\infty} d k^{\prime} \frac{\sigma_{\alpha \alpha^{(1)}}\left(k^{\prime}\right)}{k^{\prime 2}+\mu^{2}},
\end{aligned}
$$

which perhaps illustrates more clearly the choice of $\omega_{0}=0$ as reference energy.

The derivation of the dispersion relations which we have given here is based on the formalism of present day quantum field theory. It would appear, however, to make use only of the essentially minimal features of the theory, which we would abandon only with extreme reluctance, such as Lorentz invariance, and the concept of the $S$-matrix. If one were to assume that our condition on the vanishing of the commutator of the currents at space like points were too stringent, and instead should be replaced by the requirement of vanishing for space-like separations greater than a certain fundamental length, the dispersion relations would be modified in an essential manner. We shall not discuss these modifications here, but merely remark that there are complications similar to those which arise when one considers the scattering amplitudes for individual angular momenta.

\section{ACKNOWLEDGMENTS}

The derivation presented here is one whose existence was conjectured by Dr. M. Gell-Mann and the author several years ago and various aspects of this early work with Dr. Gell-Mann appear throughout the paper. The author is also indebted to Professor Y. Nambu and to Dr. H. Miyazawa and Dr. R. Oehme for many very helpful suggestions.

Note added in proof.-It has been pointed out to the author by Professor R. Karplus (private communication) that in the derivation of the dispersion relation, Eq. (3.3), we have effectively assumed that not only does $\sigma_{\alpha \alpha}{ }^{(1)}(k)$ go to zero faster than $1 / k$ for large $k$, but also that $D_{\alpha \alpha}(k)$ also approaches zero. This may be seen by comparing Eq. (3.4) with the limit as $k$ goes to infinity of Eq. (2.40), dropping the bound states. We cannot, however, exclude the possibility of $D_{\alpha \alpha}^{(1)}(k, \lambda)$ approaching a constant in which case there need be no contradiction, since $D_{\alpha \alpha_{1}}{ }^{(1)}(0)-D_{\alpha \alpha}{ }^{(1)}(\infty)$ may be positive and equal to the right-hand side of Eq. (3.4). It is the author's opinion that this is not the case and that the conclusion drawn in the text is correct. 